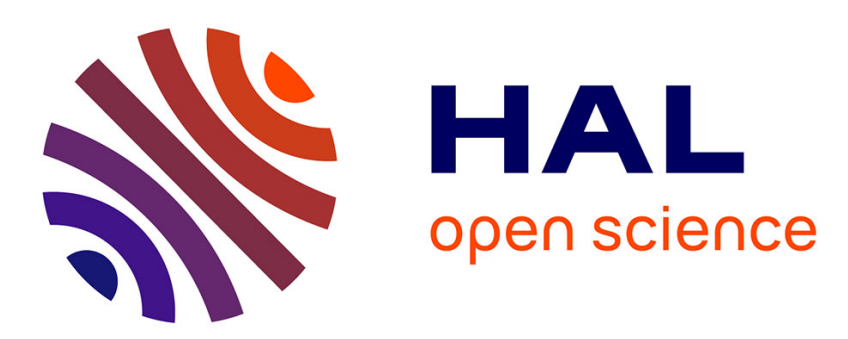

\title{
Modelling the dynamics of virus infection and immune response in space and time
}

G Bocharov, A Meyerhans, N Bessonov, S Trofimchuk, Vitaly Volpert

\section{To cite this version:}

G Bocharov, A Meyerhans, N Bessonov, S Trofimchuk, Vitaly Volpert. Modelling the dynamics of virus infection and immune response in space and time. International Journal of Parallel, Emergent and Distributed Systems, 2017, 10.1080/17445760.2017.1363203 . hal-01578907

\section{HAL Id: hal-01578907 \\ https://hal.science/hal-01578907}

Submitted on 30 Aug 2017

HAL is a multi-disciplinary open access archive for the deposit and dissemination of scientific research documents, whether they are published or not. The documents may come from teaching and research institutions in France or abroad, or from public or private research centers.
L'archive ouverte pluridisciplinaire HAL, est destinée au dépôt et à la diffusion de documents scientifiques de niveau recherche, publiés ou non, émanant des établissements d'enseignement et de recherche français ou étrangers, des laboratoires publics ou privés. 
The International Journal of Parallel, Emergent and Distributed Systems

Vol. 00, No. 00, Month 2016, 1-15

\title{
RESEARCH ARTICLE
}

\section{Modelling the dynamics of virus infection and immune response in space and time}

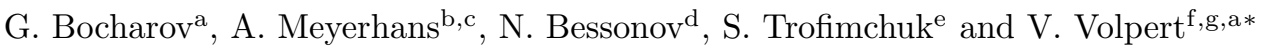 \\ ${ }^{a}$ Institute of Numerical Mathematics, Russian Academy of Sciences, Gubkina Street 8, \\ 11933 Moscow, Russia; ${ }^{\mathrm{b}}$ Infection Biology Laboratory, Department of Experimental and

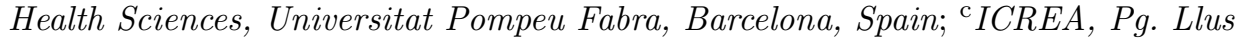 \\ Companys 23, 08010 Barcelona, Spain; ${ }^{\mathrm{d}}$ Institute of Problems of Mechanical Engineering, \\ Russian Academy of Sciences, 199178 Saint Petersburg, Russia; ${ }^{\mathrm{e}}$ Instituto de Matematica \\ y Fisica, Universidad de Talca, Casilla 747, Talca, Chile ${ }^{\mathrm{f}}$ Institut Camille Jordan, UMR \\ 5208 CNRS, University Lyon 1, 69622 Villeurbanne, France; ${ }^{\mathrm{g}}$ INRIA Team Dracula, \\ INRIA Lyon La Doua, 69603 Villeurbanne, France;
}

(v1.1 released Dec 2016)

\begin{abstract}
Spreading of viral infection in the tissues such as lymph nodes or spleen depends on virus multiplication in the host cells, their transport and on the immune response. Reactiondiffusion systems of equations with delay represent an appropriate model to study this process. The properties of immune response and the initial viral load determine the regimes of infection spreading. Infection can be completely eliminated or it can remain present together with a decreased concentration of immune cells. Finally, immune cells can be completely exhausted leading to a high virus concentration in the tissue.
\end{abstract}

Keywords: infection spreading; tissue; immune response; mathematical model; reaction-diffusion equations; time-delay

\section{Introduction}

Acute and chronic virus infections remain to be a problem of major public concern worldwide. Defence of the host organism against an infectious challenge is a primary function of the immune system associated with generation of potent responses [1]. Understanding the processes and parameters that underline fate decision between resolving and chronic dynamics of the virus infections such as CMV, HBV, HCV, HIV is a challenge. One of the major goals of systems approaches to immunology is to quantitatively measure complex multiscale spatial and temporal interactions, permitting development of computational models that can be used to predict the responses to perturbations [2]. In addition, they feed conceptual developments in theory of the immune system which due to its capability to perform many complex "computations" in a highly parallel and distribute fashion, is considered as a source of inspiration for developing intelligent methodologies for complex problem solving [3].

Although the mathematical models, like experimental animal systems [4], can not entirely reproduce the complex etiology of virus infections such as HIV in hu-

*Corresponding author. Email: volpert@math.univ-lyon1.fr 
mans or experimental LCMV infection, the modelling results might inform studies more difficult to undertake in humans. In particular, the computational models of antiviral immune response can provide insights into the regulation of the dynamics processes underlying various patterns in disease progression. Except for few studies, mathematical models of the immune response against virus infections conventionally consider the infected whole organism as a single homogenous compartment $[5,6]$. Thus they do not take into account that the dynamics of infection spread differs between tissues, organs and blood. A more detailed description however should include this spatial heterogeneity with respect to virus propagation and immune response development [7], [8].

Development of a viral infection (e.g., HIV, SIV, LCMV) in tissues such as lymph nodes or spleen occurs due to virus spreading that may be either direct between neighboring cells (cell-to cell model) or by means of a random motion of the free virus in the extracellular matrix. The infected cells are the sources of virus production. Reaction-diffusion equations represent an appropriate model for the description of virus transport and production, and of the spatiotemporal dynamics immune response that eliminates infected cells.

Interaction between the virus and the host organism includes many cellular and humoral components of the innate and adaptive arms of the immune system [9]. Virus-specific cytotoxic T lymphocytes (CTL) play a key role in the dynamics and outcome of virus infections $[10,11]$. Importantly, that CTL temporal dynamics and viral growth dynamics can under many circumstances be successfully captured using simple conceptual views of the regulation based on predator-prey systems [13], there are other phenomena, such as CTL exhaustion, which do not fit in this simple predator-prey interpretation and demand more complex conceptual models (e.g., the balance of growth and differentiation models) to capture reality [14]. Since long ago, it was observed that virus infections have a strong impact on the immune system [15].

The experimental LCMV infection in mice provided a solid evidence that the intensity of the CTL response depends on the viral load and growth kinetics in a nonlinear manner [10, 12]. A small infection level will stimulate an immune response while a sufficiently high infection level will downregulate an immune response by immune cell death and/or decrease of immune cell proliferation. Moreover the action of the immune response is delayed in time since, after the stimulation by infection, immune cells need some time to proliferate and differentiate. Thus, infection spreading in the tissue can be modelled by reaction-diffusion equations with delay.

In our previous work [6] we studied the spread of a viral infection in tissue with a single reaction-diffusion equation for the virus concentration. The concentration of the immune cells was considered as a function of the infection level. With this model different regimes of infection spreading were identified. Depending on the intensity of the immune response and the initial viral load, we observed a) infection propagation with a low viral load established behind the infection front, b) a high infection level behind the infection front, and c) two consecutive infection fronts, the first one with a low infection level and the second one behind it with a high infection level.

In this work here we now consider a more detailed model of infection spreading in tissue with a reaction-diffusion system of two equations, one for the concentration of virus and one for that of immune cells (CTLs). The model is formulated in the next section. We discuss the corresponding ordinary differential system of equations in Section 3. The results of numerical simulations of the reaction-diffusion system with delay are presented in Section 4. Finally, we discuss the results and implications of 
our analysis for understanding the pathogenesis of virus infections in Section 5.

\section{Model}

The biological scheme of the model is presented in Figure 1. In the model we consider multiple non-linear feedback regulations of the population dynamics of virus infection and the antiviral immune responses. According to this general scheme, the viruses and immune cells compete for their survival. The outcome of the competition results from the race between the virus infection spread on one side and the clonal CTL expansion followed by migration to the site of infection to eliminate the virus sources. Depending on the viral load and growth kinetics, the efficacy of the CTL response in determined by a balance of proliferation, anergy induction and activation-induced cell death by apoptosis. The later two take into account the tuning of lymphocytes [14], a key feature of the immune system.

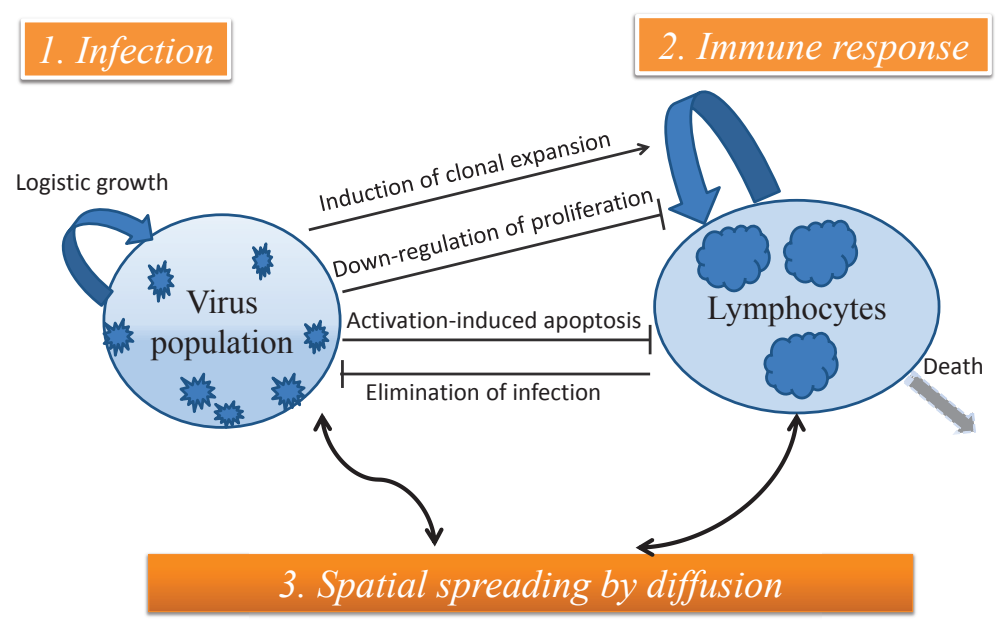

Figure 1. Schematic representation of the processes underlying the population dynamics of virus infection spread and the antiviral immune response.

\subsection{Two PDEs model}

We consider the following system of equations for the concentration of virus $v$ and of immune cells $c$ in lymphoid organs such as the spleen or lymph nodes, viewed as 1D structures:

$$
\begin{gathered}
\frac{\partial v}{\partial t}=D_{1} \frac{\partial^{2} v}{\partial x^{2}}+k v(1-v)-\sigma v c \\
\frac{\partial c}{\partial t}=D_{2} \frac{\partial^{2} c}{\partial x^{2}}+\left(c_{0}+\phi\left(v_{\tau}\right) c\right)(1-c)-\psi\left(v_{\mu}\right) c .
\end{gathered}
$$

Here $v$ and $c$ are the normalized dimensionless concentrations of virus and immune cells, respectively, $v_{\tau}=v(x, t-\tau), v_{\mu}=v(x, t-\mu)$, the functions $\phi\left(v_{\tau}\right)$ and 
$\psi\left(v_{\mu}\right)$ will be specified below. The first term in the right-hand side of equation (1) describes the virus diffusion, the second term its production and the last term its elimination by the immune cells. The parameter $D_{1}$ is the diffusion coefficient of virus and $k_{1}$ stands for the replication rate constant. Similarly, the first term in the right-hand side of the second equation describes random motion of immune cells, the second term gives the rate of the appearance of immune cells migrating to the peripheral organs from the bone marrow and thymus and proliferating there and the last term parameterizes the rate of their death. The second term includes cell influx due to differentiation of less mature precursor cells and cell birth due to antiviral immune response. The latter, depends on the viral load in a non-linear bell-shaped manner. The last term for cell death includes natural (constant) cell death and their apoptosis induced by infection. Both of them are included in the function $\psi\left(v_{\mu}\right)$. Parameters $D_{1}, D_{2}, k$ and $\sigma$ are positive numbers, the functions $\phi(v)$ and $\psi(v)$ are assumed to be positive and sufficiently smooth. The delay values in $\phi(v)$ and $\psi(v)$ represent the net duration of cell proliferation and differentiation to effector cells and the time needed for programming of activated CTLs to apoptosis, accordingly [16].

In what follows we will consider the function $f(v)=1-\psi(v) / \phi(v)$. We will suppose that $f(0)>0$, it is increasing for sufficiently small $v$ and decreasing for $v$ large enough. Such properties of this function corresponds to the biological observation that small viral load promotes proliferation of immune cells while large level of infection decreases cell proliferation and increases their death. This function can remain positive for all $v$ or it can become negative for $v$ sufficiently large. A typical form of this function is shown in Figure 2.

We note that the number of immune cells increases enormously i.e. by $10^{3}$-fold, via clonal expansion in the course of immune response to infection. Therefore, we will neglect the cell influx term in comparison with the effect of clonal proliferation on the population size and we will set $c_{0}=0$. This assumption allows us to simplify the model analysis.

\subsection{Reduction to a single PDE model}

If we consider the functions $\phi$ and $\psi$ in the form

$$
\phi(v)=R \phi_{0}(v), \quad \psi(v)=R \psi_{0}(v)
$$

where $R$ is a large parameter, then we can divide the second equation by $R$ and formally pass to the limit $R \rightarrow+\infty$. Then we get

$$
c=f(v), \text { where } f(v)=1-\frac{\psi(v)}{\phi(v)} .
$$

If $\tau=\mu$, then substituting this expression into equation (1), we get the equation

$$
\frac{\partial v}{\partial t}=D_{1} \frac{\partial^{2} v}{\partial x^{2}}+k v(1-v)-\sigma f\left(v_{\tau}\right) v
$$

introduced and studied in [6]. As it is mentioned in the introduction, there are three main regimes of the infection spreading in this case: with low level infection level behind the infection front, with high level infection level, and with low level followed 
by high level infection. The choice between them is determined by the intensity of immune response (function $f(v)$ ) and by the initial infection distribution in the tissue.

\section{Existence and stability of stationary points}

\subsection{Two ODEs version of the model}

We begin to study system (1), (2) with the stationary points of the ODE system

$$
\begin{gathered}
\frac{d v}{d t}=k v(1-v)-v c, \\
\frac{d c}{d t}=\phi(v) c(1-c)-\psi(v) c,
\end{gathered}
$$

where we set $\sigma=1$. The stationary points of this system are determined by the equations

$$
v=0 \text { or } c=k(1-v) ; \quad c=0 \text { or } c=f(v) \text {. }
$$
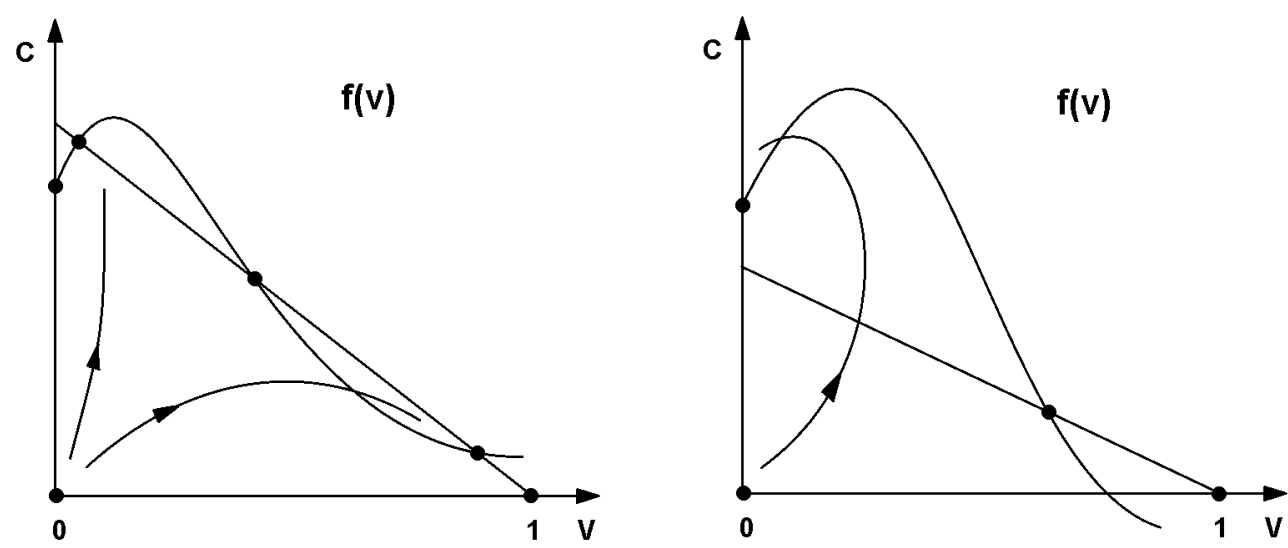

Figure 2. Schematic representation of the phase plane of system (4), (5). There are 6 stationary points in the left figure. Two of them are stable (see the explanation in the text). The function $f(v)$ becomes negative for large $v$ in the right figure. There are also two stable stationary points, the most left and the most right ones.

Some typical examples of nullclines, stationary points and trajectories of this system are shown in Figure 2. There are 6 stationary points in the left figure, $P_{1}=$ $(0,0), P_{2}=(0, f(0)), P_{i}=\left(v_{i}, c_{i}\right), i=3,4,5,6$, where $0<v_{3}<v_{4}<v_{5}<v_{6}=1$, $0=c_{6}<c_{5}<c_{4}<c_{3}$. Stability of these points are determined by the eigenvalues of the matrix

$$
A=\left(\begin{array}{cc}
k(1-2 v)-c & -v \\
\phi^{\prime}(v) c(1-c)-\psi^{\prime}(v) c \phi(v)(1-2 c)-\psi(v)
\end{array}\right)
$$


It can be easily verified that $P_{1}$ and $P_{6}$ are saddle points, $P_{2}$ is also a saddle if $k>f(0)$ (Figure 2, left) and it is a stable node if $k<f(0)$ (Figure 2, right). The other points with positive coordinates are stabile if $f^{\prime}\left(v_{i}\right)>-k$ and unstable if the inequality is opposite.

Depending on the values of parameters, the trajectory starting in the vicinity of the unstable point $P_{1}$ can have one of the following behaviors.

1. If $f(0)<k$, then the point $P_{3}(0, f(0))$ is stable (Figure 2 , left). Since $v_{3}>0$, then virus is not eliminated. This case corresponds to chronic infection with a low viral load. The level of virus infection can monotonically increase in time converging to its limiting value $v_{3}$ or it can pass over its maximal value and decrease towards $v_{3}$.

2. If $f(0)>k$, then the point $P_{2}(0, f(0))$ is stable, and the trajectory can converge to it for large time (Figure 2, right). This case corresponds to complete virus elimination after acute infection. The memory immune cells remain in the body providing rapid immune response in the case of further infections.

3. Another stable stationary point is $P_{5}=\left(v_{5}, c_{5}\right)$, where $f^{\prime}(v)>-1$. The corresponding value of $v$ is large and of $c$ low. This is the case of infection induced immunodeficiency characterized by high viral load and partial exhaustion of CTLs.

4. If $f(v)<0$ for $v=1$ (Figure 2, right), then the stable stationary point $v=$ $1, c=0$ corresponds to complete exhaustion of immune cells.

\subsection{Two Delay Differential Equations version of the model}

In this section we study stability of the same stationary points of the ODE system with the delays introduced into the corresponding terms as in the original PDE model (1)-(2):

$$
\begin{gathered}
\frac{d v}{d t}=k v(1-v)-v c, \\
\frac{d c}{d t}=\phi\left(v_{\tau}\right) c(1-c)-\psi\left(v_{\mu}\right) c .
\end{gathered}
$$

Let us note that a similar system with particular form of the functions $\phi(v)$ and $\psi(v)$ was studied in [16]. In order to analyze below behavior of solutions of the reaction-diffusion system, we begin with the study stability of the stationary solution $\left(v_{*}, c_{*}\right)$ with respect to this DDEs system with two delays. After linearization about this stationary solution, we obtain the equation with respect to the eigenvalue $\lambda$ :

$$
\operatorname{det}(A(\lambda)-\lambda E)=0
$$

where

$$
A(\lambda)=\left(\begin{array}{cc}
-k v_{*}, & -v_{*} \\
c_{*} g(\lambda), & -c_{*} \phi\left(v_{*}\right)
\end{array}\right), \quad g(\lambda)=\phi^{\prime}\left(v_{*}\right)\left(1-c_{*}\right) e^{-\lambda \tau}-\psi^{\prime}\left(v_{*}\right) e^{-\lambda \mu} .
$$

We recall that 


$$
c_{*}=k\left(1-v_{*}\right), \quad \phi\left(v_{*}\right)\left(1-c_{*}\right)-\psi\left(v_{*}\right)=0 .
$$

Equation (9) can be written as follows:

$$
\phi(v) \frac{(k v+\lambda)(k(1-v) \phi(v)+\lambda)}{k v(1-v)}=\phi(v) \psi^{\prime}(v) e^{-\lambda \mu}-\phi^{\prime}(v) \psi(v) e^{-\lambda \tau}
$$

( $v_{*}$ is replaced here by $v$ for simplicity of notation). In order to find the onset of oscillatory instability, we set $\lambda=i \xi$. Equating the real and imaginary parts, we get:

$$
\begin{gathered}
\frac{\phi(v)}{k v(1-v)}\left(k^{2} v(1-v) \phi(v)-\xi^{2}\right)=\phi(v) \psi^{\prime}(v) \cos (\xi \mu)-\phi^{\prime}(v) \psi(v) \cos (\xi \tau), \\
\frac{\phi(v)}{v(1-v)}(v+(1-v) \phi(v)) \xi=-\phi(v) \psi^{\prime}(v) \sin (\xi \mu)+\phi^{\prime}(v) \psi(v) \sin (\xi \tau),
\end{gathered}
$$

For a given $v$ (stationary point, equations (10)) and $\tau$ we obtain a system of equations with respect to $\xi$ and $\mu$. The value of $\mu$ determines the stability boundary and the value of $\xi$ the period of oscillations.
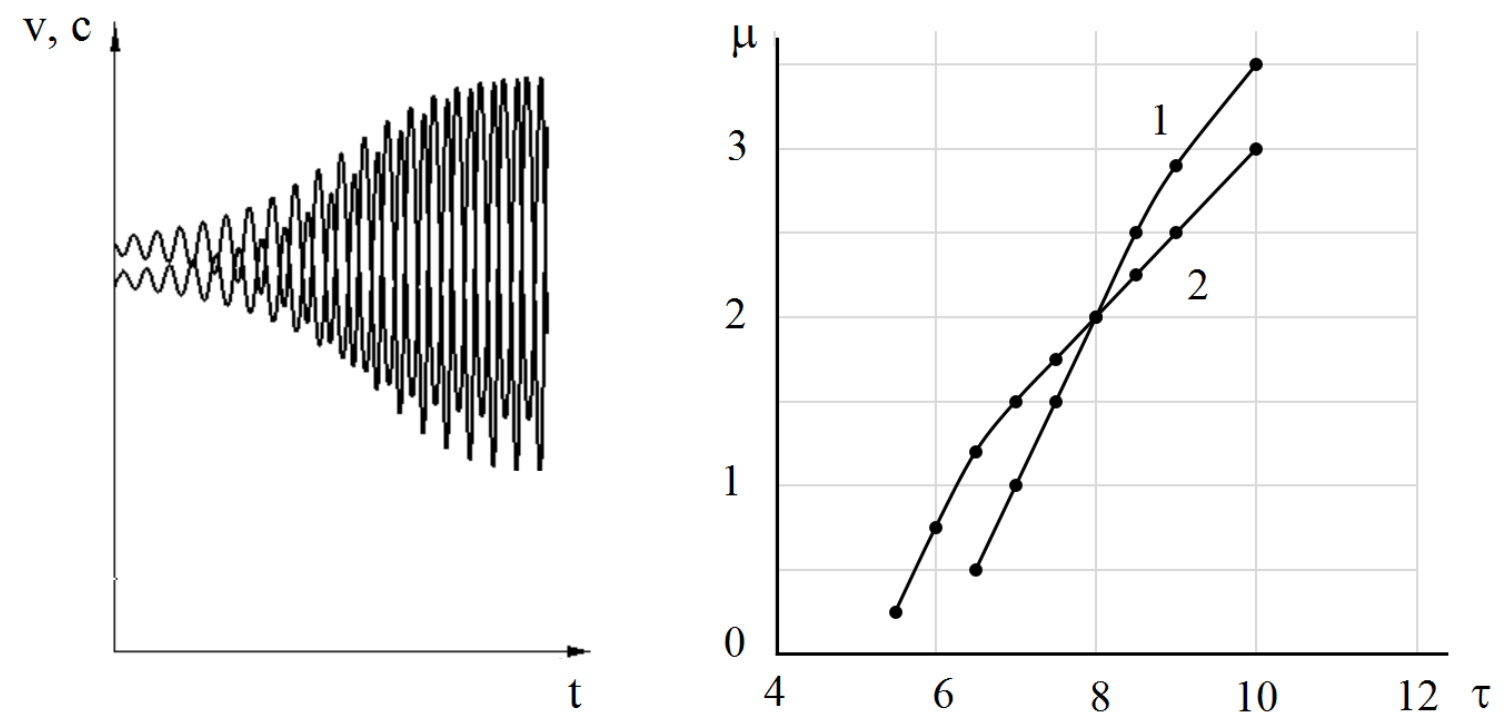

Figure 3. Example of periodic oscillations for the values of parameters $k=\sigma=1, \phi(v)=2 v, \psi(v)=v$, $\tau=10, \mu=2$ (left). Stability boundary on the $(\tau, \mu)$ plane for $k=\sigma=1$ (right): Curve 1 is defined by $\phi(v)=3 v, \psi(v)=v$, and curve 2 by $\phi(v)=2 v, \psi(v)=v$. The stability region is located above the curves.

Example of numerical simulations and stability boundary on the $\tau, \mu$ plane are shown in Figure 3. It is interesting to note that the first time delay, $\tau$, destabilizes the solution while the second one, $\mu$, stabilizes it. 


\section{Reaction-diffusion system with delays}

Behavior of solutions of system (1), (2) depends on the functions $\phi(v)$ and $\psi(v)$ which determine production and death of immune cells. We will consider some particular cases below.

\subsection{Constant $\phi$, linear $\psi$}

If we assume $\phi(v) \equiv \phi_{0}$ (see, e.g. [17]) and $\psi(v)=\psi_{0} v$ reflecting the loss of CTLs on elimination of infection, then for $c_{0}=0$ we obtain the system

$$
\begin{gathered}
\frac{\partial v}{\partial t}=D_{1} \frac{\partial^{2} v}{\partial x^{2}}+k v(1-v)-\sigma v c, \\
\frac{\partial c}{\partial t}=D_{2} \frac{\partial^{2} c}{\partial x^{2}}+\phi_{0} c(1-c)-\psi_{0} v_{\mu} c .
\end{gathered}
$$

The initial condition is considered in the form

$v(x, t)=0, c(x, t)=0.5, \quad 0<x<L / 2 ; \quad v(x, t)=0.5, c(x, t)=0, \quad L / 2<x<L$,

where $L$ is the length of the computational interval, $-\max (\tau, \mu) \leq t \leq 0$. We consider the no-flux boundary conditions for both variables.

In the case without delay $(\mu=0)$ system $(14),(15)$ is similar to the well known system of competition of species in population dynamics. We will consider it also on the whole axis in order to study travelling wave solutions.
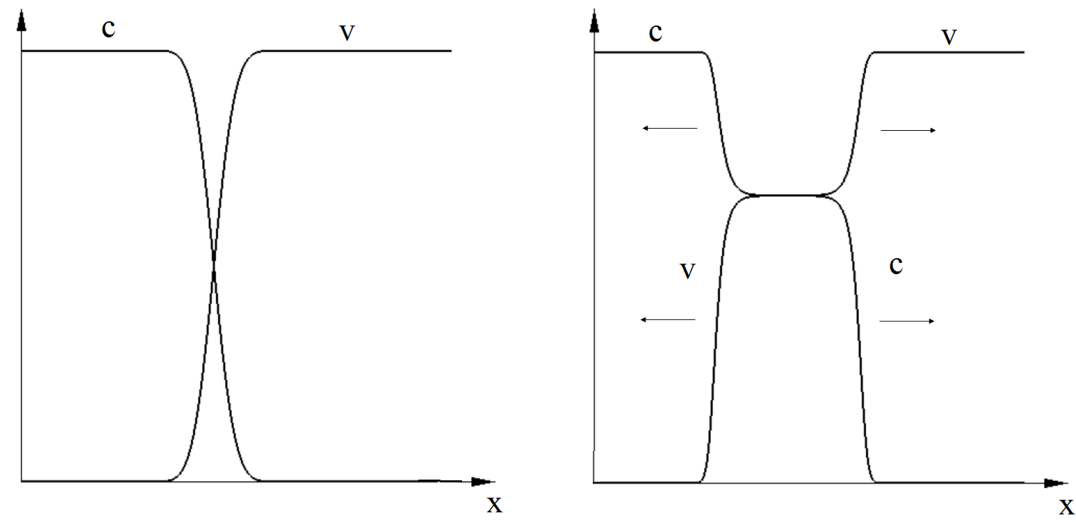

Figure 4. The values of parameters: $D_{1}=D_{2}=10^{-5}, k=\sigma=1, \phi_{0}=1, \psi_{0}=1 ; \tau=\mu=1$ (left), $\sigma=0.5, \psi_{0}=0.5$ (right).

The corresponding DDE system (without diffusion) has four stationary points, $P_{0}=(0,0), P_{1}=(1,0), P_{2}=(0,1)$, and $P_{3}=\left(v_{*}, c_{*}\right)$, where the coordinate of the last point are positive and they can be easily found from the linear algebraic system of equation. The point $P_{0}$ is unstable as a solution of the DDE system, points $P_{1}$ and $P_{2}$ can be stable provided that $P_{3}$ is unstable and vice versa. Here and in what follows we say that the point is stable if all its eigenvalues have negative real parts, and that it is unstable if one of the eigenvalues has a positive real part. 
Consider first the case where the points $P_{1}$ and $P_{2}$ are stable and the point $P_{3}$ is unstable. Then there exist travelling wave solutions of system (14), (15), that is the solution $v(x, t)=V(x-s t), c(x, t)=C(x-s t)$, where $s$ is the speed of propagation and the functions $V, C$ satisfy the system of equations

$$
\begin{gathered}
D_{1} V^{\prime \prime}+s V^{\prime}+k^{\prime} V(1-V)-\sigma V C=0, \\
D_{2} C^{\prime \prime}+s C^{\prime}+\phi_{0} c(1-c)-\psi_{0} V(x+s \mu) C=0
\end{gathered}
$$

with the conditions

$$
C(-\infty)=1, C(\infty)=0, \quad V(-\infty)=0, V(\infty)=1
$$

at infinities. Existence of such solution is known for the case without delay $(\mu=0)$ [18] and for a single equation with delay (see [6] and the references therein). We expect that the existence result remains valid for the system of two equations with delay since the methods based on maximum principle remain applicable here.

The speed of the wave can be positive, negative or zero depending on the values of parameters. Figure 4 (left) shows the wave with the zero speed, in other words a stationary solution of system (14), (15). The speed remains zero in a large interval of variation of time delay $\mu$.

Proposition 3.1. Let $s_{\mu}$ be the speed of the travelling wave of system (14), (15). If $s_{0}>0\left(s_{0}<0\right)$, then $s_{\mu}>0\left(s_{\mu}<0\right)$ for all $\mu \geq 0$.

Proof. For the proof of this assertion it is sufficient to note that $s_{\mu}$ is a continuous function of $\mu$. Therefore if $s_{0}>0$ and $s_{\mu_{0}}<0$ for some $\mu_{0}>0$, then $s_{\mu}=0$ for some $0<\mu<\mu_{0}$. Hence system (16), (17) does not contain delay. The speed of the wave, since it is unique, becomes $s_{\mu}=s_{0}>0$. This contradiction proves the proposition.

Next, consider the case where the point $P_{3}$ is stable while the points $P_{1}$ and $P_{2}$ are unstable. In the case without delay it is known [18] that system (16), (17) have solutions with the limits

$$
C(-\infty)=1, C(\infty)=c_{*}, \quad V(-\infty)=0, V(\infty)=v_{*}
$$

for $s \leq s_{-}$, and with the limits

$$
C(-\infty)=c_{*}, C(\infty)=0, \quad V(-\infty)=v_{*}, V(\infty)=1
$$

for $s \geq s_{+}$. Here $s_{+}$is some positive and $s_{-}$some negative values. Numerical simulations show that such solutions also exist for a positive time delay $\mu$ (Figure 4 , right). The absolute values $\left|s_{ \pm}\right|$increase as a function of $\mu$.

\subsection{Linear $\phi$ and $\psi$}

We now consider linear functions $\phi$ and $\psi: \phi(v)=\phi_{1} v$ and $\psi(v)=\psi_{1} v$. The system becomes as follows: 


$$
\begin{gathered}
\frac{\partial v}{\partial t}=D_{1} \frac{\partial^{2} v}{\partial x^{2}}+k v(1-v)-\sigma v c, \\
\frac{\partial c}{\partial t}=D_{2} \frac{\partial^{2} c}{\partial x^{2}}+\phi_{1} v_{\tau} c(1-c)-\psi_{1} v_{\mu} c .
\end{gathered}
$$

The corresponding DDE system has the following non-negative stationary points:

$$
v=0, c \geq 0 ; \quad v=1, c=0 ; \quad v_{*}=1-\frac{\sigma}{k}\left(1-\frac{\psi_{1}}{\phi_{1}}\right), c_{*}=1-\frac{\psi_{1}}{\phi_{1}} .
$$

We consider the same initial conditions as before. For the values of parameters indicated in the caption to Figure 5 the stationary point $\left(v_{*}, c_{*}\right)$ with the positive coordinates is stable for sufficiently small time delays $\tau$ and $\mu$. In this case, we observe two travelling waves. The first one with the negative speed provides the transition from the point $v=0, c=0.5$ (determined by the initial condition) to the point $\left(v_{*}, c_{*}\right)$, and the second one with the positive speed transition from $\left(v_{*}, c_{*}\right)$ to $v=1, c=0$ (Figure 5, left). The interval where the solution converges to the positive equilibrium grows in time.
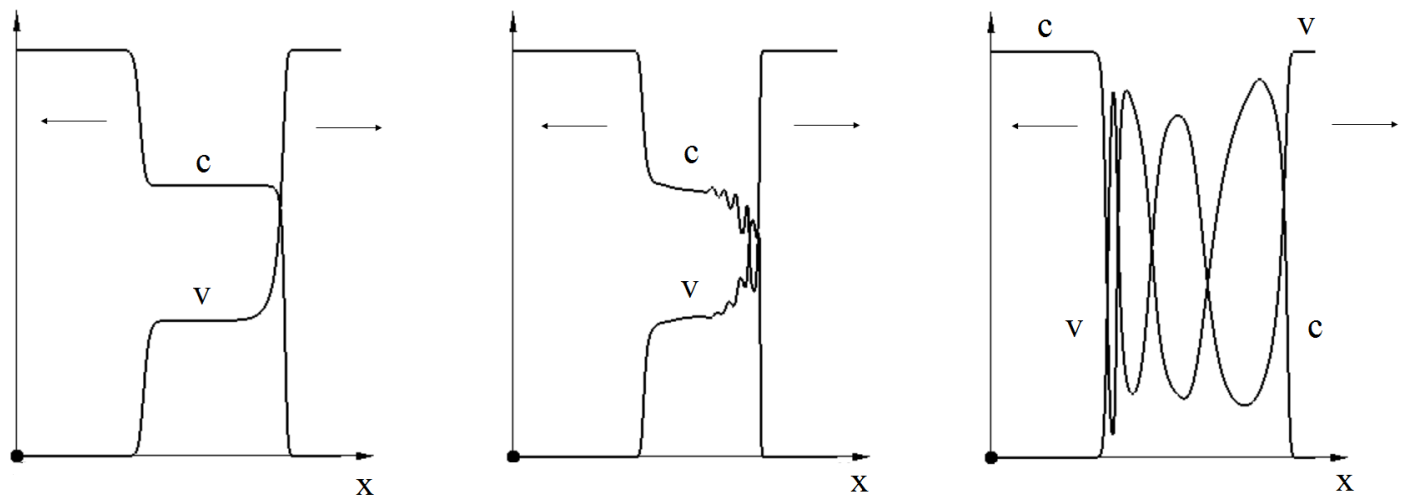

Figure 5. The values of parameters: $D_{1}=D_{2}=10^{-5}, k=\sigma=1, \phi(v)=3 v, \psi(v)=v ; \tau=\mu=1$ (left), $\tau=1, \mu=10, D_{1}=D_{2}=10^{-6}$ (middle), $\tau=10, \mu=1$ (right).

Behavior of solutions depends on the values of time delays. For $\mu=10, \tau=$ 1 , the stationary point $\left(v_{*}, c_{*}\right)$ remains stable but there are decaying oscillations behind the wave fronts (Figure 5, middle. Decreased diffusion coefficients shorten the space oscillations). If $\mu=1, \tau=10$, then this point is unstable and we observe spatiotemporal oscillations between the two waves (Figure 5, right). If both time delays are large and equal to each other, $\mu=10, \tau=10$, then the stationary point becomes again stable.

\subsection{Bell-shaped $\phi$ and linear $\psi$}

Finally we will consider a a most general bell-shaped function $\phi(v)$ describing specifically the induction and down-regulation of immune response and a linear function $\psi(v)=\psi_{2} v$ parameterizing the effect of viral population on death of immune cells: 


$$
\begin{gathered}
\frac{\partial v}{\partial t}=D_{1} \frac{\partial^{2} v}{\partial x^{2}}+k v(1-v)-\sigma v c, \\
\frac{\partial c}{\partial t}=D_{2} \frac{\partial^{2} c}{\partial x^{2}}+\phi\left(v_{\tau}\right) c(1-c)-\psi_{2} v_{\mu} c .
\end{gathered}
$$

The first three stationary points of the corresponding ODE system are the same as in the case where both functions are linear (Section 3.1): $P_{0}=(0,0), P_{1}=$ $(1,0), P_{2}=(0,1)$. The point $P_{3}=\left(v_{*}, c_{*}\right)$ with positive coordinates can be found from the algebraic system of equations:

$$
c=\frac{k}{\sigma}(1-v), \quad c=1-\frac{\psi_{2} v}{\phi(v)} .
$$

Note that, its solution can be non-unique. Moreover, stability of stationary points $P_{1}$ and $P_{2}$ versus $P_{3}$ is not mutually exclusive, as it was before.
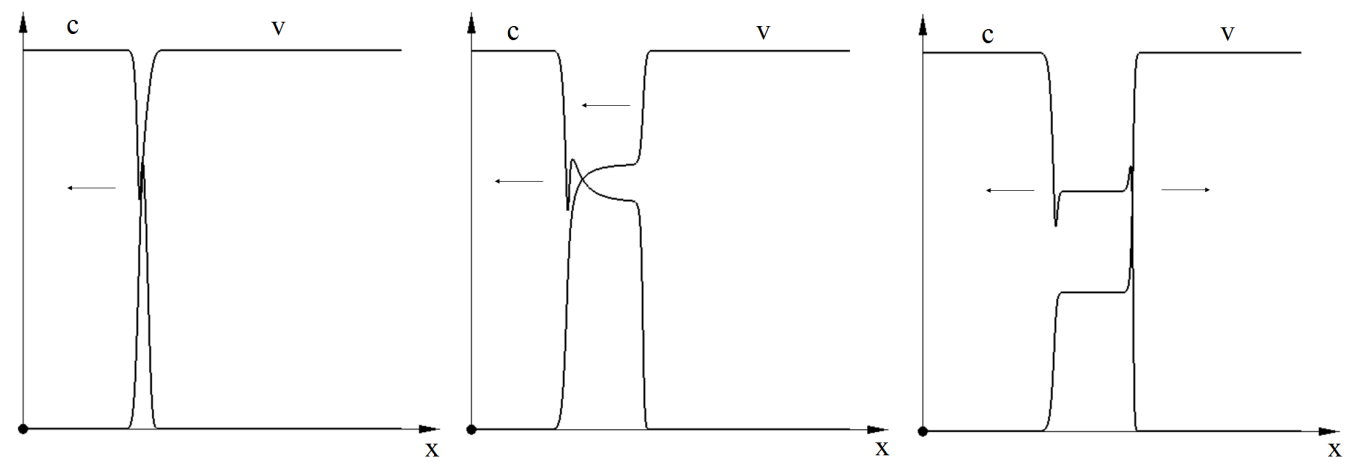

Figure 6. The values of parameters: $D_{1}=D_{2}=10^{-5}, k=1, \psi(v)=5 v ; \tau=\mu=1 ; \sigma=0.2$ (left), $\sigma=0.5$ (middle), $\sigma=1$ (right).
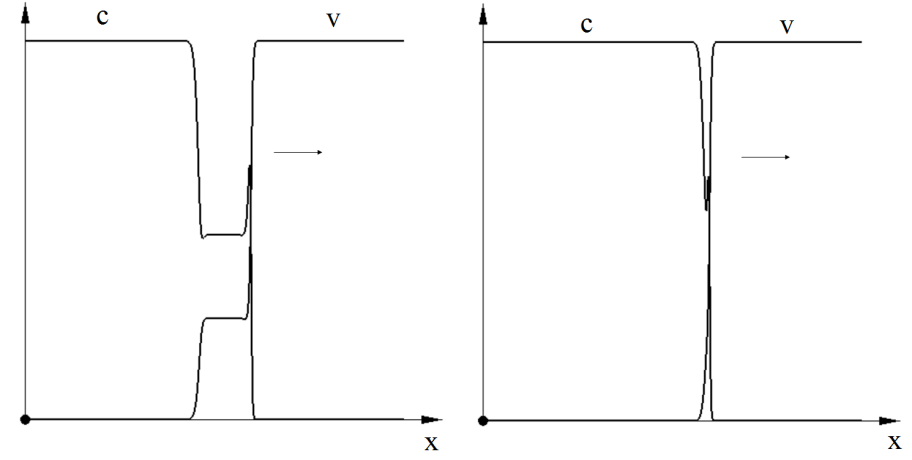

Figure 7. The values of parameters: $D_{1}=D_{2}=10^{-5}, k=1, \psi(v)=5 v ; \tau=\mu=1 ; \sigma=1.5$ (left), $\sigma=2$ (right).

Figures 6 and 7 show different regimes observed in numerical simulations under variation of the parameter $\sigma$, specifying the per capita elimination rate of the infection by CTLs and all other parameters fixed. For small values of $\sigma$, the virus elimination is not sufficient to stop its propagation, and a single wave with a negative velocity and with the limits 


$$
v(-\infty)=0, c(-\infty)=1 ; \quad v(\infty)=1, c(+\infty)=0
$$

is observed (Figure 6, left). For a larger value of $\sigma$, there are two consecutive waves with negative speed propagating one after another (Figure 6, left). The distance between them grows with time. For $\sigma=1$, the two waves move in the opposite directions (Figure 6, right) Further increase of this parameter leads to the appearance of two consecutive waves with positive speeds (Figure 7, middle). Finally, if it is sufficiently large, there is a single wave with a positive speed (Figure 7 , right). It is remarkable that regimes with two consecutive waves propagating with different speeds are specific for the bell-shaped function $\phi(v)$, they were not observed above (Section 3.2).
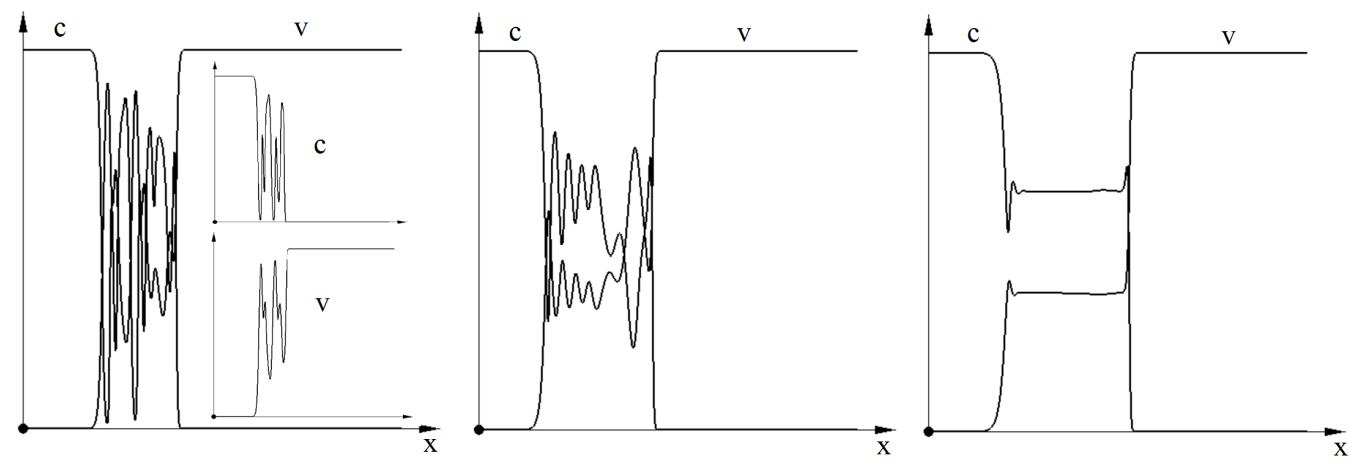

Figure 8. The values of parameters: $D_{1}=D_{2}=10^{-5}, k=\sigma=1, \psi(v)=5 v ; \tau=10, \mu=1$ (left); $\tau=1, \mu=5$ (middle), $\tau=10, \mu=10$ (right).

Time delay influences the wave structure through a possible instability of the steady state with positive coordinates. We take, as example, the result presented in Figure 6 (right) and vary the values of $\tau$ and $\mu$. As before, there is a wave with a negative speed and a wave with a positive speed. For $\tau=10, \mu=1$ they are separated by a growing zone of spatiotemporal oscillations (Figure 8 , left). The wave with a negative speed is a solution of the system

$$
\begin{gathered}
D_{1} V^{\prime \prime}+s V^{\prime}+k V(1-V)-\sigma V=0, \\
D_{2} C^{\prime \prime}+s C^{\prime}+\phi(V(x+s \tau)) C(1-C)-\psi_{2} V(x+s \mu) C=0
\end{gathered}
$$

with $s<0$ and the limits

$$
V(-\infty)=0, C(-\infty)=1 ; \quad \text { oscillating solution at }+\infty
$$

The wave with the positive speed is a solution of system (26), (27) with $s>0$ and

$$
\text { oscillating solution at }-\infty ; \quad V(\infty)=1, C(\infty)=0 .
$$

For $\mu=5$ the amplitude of the oscillations decreases, and for $\mu=10$ they completely disappear. 


\section{Discussion}

In this work we study spreading of viral infection in the tissues such as lymph node or spleen. Note that the results of our study can be extrapolated to other tissues as the governing equations remain generally valid. The model consists of a reactiondiffusion system of equations with two delays describing the population dynamics of virus and immune cells. The corresponding ODE and DDE systems have different equilibria including a virus free state, which can be stable or unstable, and a disease state where a high virus concentration can be accompanied by a decreased of completely exhausted population of immune cells. The values of parameters and the initial conditions determine the convergence to one of these equilibria.

Virus transport in the tissue plays an important role in the disease development [19], [20]. Taking it into account by formulation a reaction-diffusion model, we observe the existence of multiple steady-states and possible transition between the different equilibria. Similar to the study based on a single PDE model [6], behavior of the system is determined by the characteristics of antiviral immune response and by the initial viral load.

Proliferation and death of immune cells depends on the infection level. In order to simplify the analysis of the model, we suppose throughout this work a linear dependence of cell death on the concentration of virus. Contrary to this assumption, the dependence of cell proliferation on virus concentration is considered in three different forms. We begin with the simplest case where the proliferation rate does not depend on virus concentration reflecting the model of LCMV infection identified in [17]. In this case the studied model is similar to the system of competition of species where there are three main types of behavior of solutions:

- (i) The infection can spread in the tissue leading to a complete elimination of immune cells (exhaustion);

- (ii) The infection can be completely eliminated (healing);

- (iii) There is a stable equilibrium in which virus population co-exists with a population of virus-specific immune cells.

Importantly, in (i) and (ii) the solutions represent a travelling wave propagating either with positive or with negative speed. In the last case (iii), the system converges to the steady-state with an intermediate viral load which can be interpreted as a chronic infection.

Next, we consider the case where the proliferation rate of the immune cells is a linear function of the viral load reflecting another mode of immune response regulation as detailed in [17]. The main difference with the previous case is that the coexisting equilibrium can lose its stability because of the delay in the immune response development. The loss of stability leads to the emergence of spatiotemporal structures. The second time delay in the death term of immune cells is hown that have a stabilizing effect on the steady state.

Finally, the last case is most interesting and biologically realistic. The proliferation rate is represented by a bell shaped function of the virus concentration. It increases for small concentrations and decreases if the concentration is sufficiently high. We observe here two novel regimes not observed before. Infection propagation in the tissue can occur as a superposition of two travelling waves: first wave propagates as a low level infection front followed by a high level infection front with a smaller speed of propagation. Both of the travelling waves can have a positive or a negative speed corresponding to the infection advancement or retreat. As before, these regimes can be accompanied by instabilities and the emergence of spatiotemporal patterns. 
Understanding the regularities in the spatio-temporal dynamics of virus infections in host organisms is highly important for improving the clinical practice (e.g. infection fate regulation, biopsy procedures) and in immune inspired engineering (e.g., pattern recognition, classification, control of network systems). There are rather few models with describe the population dynamics of viruses and immune cell using distributed parameter system such as reaction-diffusion equations [6]. In this work, we have analyzed the system of reaction-diffusion PDEs by a combination of analytical and numerical studies. The model is novel in terms of the complexity of the reaction terms describing in a parsimonious way the feedback regulation of activation-, responsiveness tuning-, and apoptosis of immune cells. Our model goes beyond a common view of the virus and immune cell interactions as a predator prey system $[13,21]$.

A central problem of infectious disease pathogenesis is related to the mechanisms of $\mathrm{T}$ cell exhaustion, associated with various outcomes of infections. Mathematical models assist in revealing a mechanistic view of the infection course and outcomes $[12,13]$. In contrast to previous studies of exhaustion phenomenon, we have shown that in chronic infections the dynamics of virus and immune cells in space and time can be a continuum of different regimes ranging from steady states with a complete or a partial elimination of immune cells on one sisde to non-monotone dynamics in the form of travelling waves and irregular oscillations on the other side. The timedelays reflecting the clonal expansion of cells and the programming for apoptosis appear to be key control parameters. The mapping of the parameter space on the patters of spatio-temporal dynamics being a subject of our study can further assist in resolving via a multi-disciplinary approaches a fundamental problem of how to reverse $\mathrm{T}$ cell exhaustion in chronic virus infections [22-24].

\section{Acknowledgement}

This work was supported by the Russian Science Foundation under Grant [number 15-11-00029].

\section{References}

[1] Qi H, Kastenmller W, Germain RN. Spatiotemporal basis of innate and adaptive immunity in secondary lymphoid tissue. Annu Rev Cell Dev Biol. 2014;30:141-67.

[2] Subramanian N, Torabi-Parizi P, Gottschalk RA, Germain RN, Dutta B. Network representations of immune system complexity. Wiley Interdiscip Rev Syst Biol Med. 2015;7(1):13-38.

[3] Dasgupta, D., (Ed.), 1999. Artificial Immune Systems and Their Applications. University of Arizona, Springer-Verlag Berlin Heidelberg. ISBN 3-540-64390-7.

[4] Vrisekoop N, Mandl JN, Germain RN. Life and death as a T lymphocyte: from immune protection to HIV pathogenesis. J Biol. 2009;8(10):91.

[5] Cappuccio A, Tieri P, Castiglione F. Multiscale modelling in immunology: a review. Brief Bioinform. 2016;17(3):408-18.

[6] Bocharov G, Meyerhans A, Bessonov N, Trofimchuk S, Volpert V. Spatiotemporal dynamics of virus infection spreading in tissues. PLoS ONE. 2016;11(12):e0168576.

[7] Bouchnita A, Bocharov G, Meyerhans A, Volpert V. Hybrid approach to model the spatial regulation of $\mathrm{T}$ cell responses. BMC Immunology. 2017 (in press). 
[8] Bouchnita A,Bocharov G, Meyerhans A, Volpert V. Towards a Multiscale Model of Acute HIV Infection. Computation. 2017 (accepted)

[9] Zinkernagel RM, Hengartner H. On immunity against infections and vaccines: credo 2004. Scand J Immunol. 2004;60(1-2):9-13.

[10] Moskophidis D, Lechner F, Pircher H, Zinkernagel RM. Virus persistence in acutely infected immunocompetent mice by exhaustion of antiviral cytotoxic effector T cells. Nature. 1993;362(6422):758-61.

[11] McMichael AJ, Borrow P, Tomaras GD, Goonetilleke N, Haynes BF. The immune response during acute HIV-1 infection: clues for vaccine development. Nat Rev Immunol. 2010;10(1):11-23.

[12] Bocharov G, Argilaguet J, Meyerhans A. Understanding Experimental LCMV Infection of Mice: The Role of Mathematical Models. J Immunol Res. 2015;2015:739706.

[13] Nowak MA, May RM. Virus Dynamics. Mathematical Principles of Immunology and Virology 2000, Oxford: Oxford University Press.

[14] Grossman Z, Paul WE. Dynamic tuning of lymphocytes: physiological basis, mechanisms, and function. Annu Rev Immunol. 2015;33:677-713.

[15] Notkins AL, Mergenhagen SE, Howard RJ. Effect of Virus Infections on the Function of the Immune System. Annual Review of Microbiology. 1970;24:525538.

[16] Bocharov GA. Modelling the dynamics of LCMV infection in mice: conventional and exhaustive CTL responses. J Theor Biol. 1998;192:283-308.

[17] Baker CTH, Bocharov GA, Ford JM, Lumb PM, Norton SJ, Paul CAH, Junt T, Krebs P, Ludewig B. Computational approaches to parameter estimation and model selection in immunology. J Comput Appl Math. 2005;184:5076.

[18] Volpert A, Volpert Vit, Volpert Vl. Traveling wave solutions of parabolic systems. Translation of Mathematical Monographs. Vol. 140. Amer. Math. Society, Providence; 1994.

[19] Boulle M, Muller TG, Dahling S, Ganga Y, Jackson L, Mahamed D, Oom L, Lustig G, Neher RA, Sigal A. HIV Cell-to-Cell Spread Results in Earlier Onset of Viral Gene Expression by Multiple Infections per Cell. PLoS Pathog. 2016;12(11):e1005964.

[20] Mothes W, Sherer NM, Jin J, Zhong P. Virus Cell-to-Cell Transmission. J. Virology. 2010;84(17):83608368.

[21] Bell GI. Predator-prey equations simulating an immune response. Math Biosci. 1973;16:291314.

[22] Rowland-Jones S, de Silva T. Resisting immune exhaustion in HIV-1 infection.PLoS Med. 2008;5(5):e103.

[23] Crawford A, Angelosanto JM, Kao C, Doering TA, Odorizzi PM, Barnett BE, Wherry EJ. Molecular and transcriptional basis of CD4 T cell dysfunction during chronic infection.Immunity. 2014;40(2):289-302.

[24] Speiser DE, Utzschneider DT, Oberle SG, Mnz C, Romero P, Zehn D. T cell differentiation in chronic infection and cancer: functional adaptation or exhaustion? Nat Rev Immunol. 2014;14(11):768-74. 